\title{
Study of the psychological characteristics of the personality of students with varying degrees of severity of Internet addiction
}

\author{
Anna Kukulyar ${ }^{1}$, Anastasia Kolenova ${ }^{1}$, Oleg Butenko ${ }^{2}$, and Vera Butenko ${ }^{2}$ \\ ${ }^{1}$ Rostov Don State Technical University, 344000, Rostov-on-Don, Russia \\ ${ }^{2}$ Saint Petersburg Academy of the Investigative Committee, 190000, Saint Petersburg, Russia
}

\begin{abstract}
Nowadays, the Internet has a serious impact on the human psyche, and this problem is only gaining momentum. During the COVID19 pandemic, as part of a forced necessity, the whole world moved to the global information space. There are both pros and cons to such absolute digitalization. We, as psychologists, will be interested in the other side of the coin. On the basis of this, we conducted an empirical study dedicated to the study of the psychological characteristics of the personality of students with varying degrees of severity of Internet addiction. This article will present the results of a study involving 250 students from various universities in Russia. In the course of empirical research, we used the following methods: an author's questionnaire to determine the amount and quality of time spent by respondents on the Internet; Diagnostics of the tendency to 13 types of addictions (G.V. Lozovaya); Test "Self-assessment of mental states" (G.Yu. Eysenck); Methodology "Diagnostics of typologies of psychological defense" (R. Plutchikas adapted by L.I. Wasserman, O.F. Eryshev, E.B. Klubova, etc.); Methodology for «Determining stress resistance and social adaptation» of Holmes and Rage; Kimberly Young «Internet Addiction Test». The results obtained in the course of the research can be used by practicing psychologists, psychotherapists, clinical psychologists when working with addicts and coaddicts. The presented results can be applied in the practice of the educational process as prevention of Internet addiction.
\end{abstract}

\section{Introduction}

According to the latest relevant psychological research, the main reason for Internet addiction is compensation for low self-esteem of the user, and not a problem in communication, as it was previously believed, since this group of people does not consider the network a source of social support, as well as salvation from their loneliness $[1,2,4,9$, $10,11]$.

People tend to deny its existence or leave it instead of solving problems. As a result, the Internet user does not solve his problems, but generates new ones by his denial or escape from reality $[5,9,18]$.

\footnotetext{
*Corresponding author: vetkina-anna@mail.ru
} 
K. Young identifies 4 signs by which one can determine Internet addiction: 1) Constant desire to check mail; 2) Obsessive desire to constantly access the Internet; 3) Excessive spending time on the Internet; 4) Excessive waste of money on the Internet [16,17].

V. A. Burova, following Young, K. S., Weinstein A., in his work "Internet addiction as a form of non-chemical addictive disorder", identifies some causes of addiction: anonymity on the Web-the user experiences a sense of security and security; new (additional) images of the user are formed (his various fantasies); wide opportunities for finding new partners by correspondence, you can also easily abandon it and look for a new one [2].

In turn, Internet addiction is a harmful non-chemical addiction that is observed in Internet users. Translated from English, it means vicious, painful addictions and are called addiction, hence the term "addictive behavior". The very term "addiction" began to be used as equivalent to the term "addiction".

Note that addictive behavior is a departure from reality by means of a person changing his mental state, that is, the reality created by a computer, obviously, changes the mental state, and this is precisely what individuals prone to Internet addiction have begun to use. The computer has become a fetish, that is, a part of this or that ritual, has replaced drugs, gambling, etc. A computer user prone to Internet addiction, when using it, is in an altered state of consciousness - a psychological trance $[1,9,6,4]$.

A. Goldberg put forward his own system of criteria for Internet addiction. In his opinion, Internet addiction can be defined by three points: 1) the amount of time needed by the user to achieve satisfaction increases; 2) if a person does not increase the amount of time he spends on the Internet, then the effect is noticeably reduced; 3 ) if the user stops or shortens the time spent on the Internet, then this leads to poor health, which develops in different intervals, for example, from several days to a month and is very often expressed by more than two factors, such as emotional and motor excitement, increased anxiety, obsessive thoughts about the Internet, as well as voluntary and non-voluntary finger movements [5].

A.E. Voiskunsky in turn identifies 6 types of Internet addiction: 1) Gambling addiction; 2) Addiction to watching movies over the Internet; 3) Obsessive web surfing; 4) Cybersex addiction; 5) Addiction to virtual communication and virtual dating, 6) Obsessive financial need (Internet auctions or casinos) [12].

A number of factors were also identified with the help of which the Internet becomes attractive (as a means of escaping reality and getting pleasure), that is, an addictive agent that involves going into virtual reality (life on the Internet replaces reality and the Internet user compensates for his needs, and that's all for him. succeeds). The Internet replaces real life, and this is especially often the case for those people who are lonely for various reasons. Since the Internet makes it possible to search for a new interlocutor who will be suitable for any criteria (goals, interests, needs).

Based on the foregoing, we can conclude that in our time the Internet has a serious impact on the human psyche and this problem is extremely urgent. On the basis of this, we conducted an empirical study dedicated to the study of the psychological characteristics of the personality of students with varying degrees of severity of Internet addiction.

\section{Research methods and techniques}

The empirical study involved 250 students from various universities in Russia. In the course of the study, we used the following methods: an author's questionnaire to determine the amount and quality of time spent by respondents on the Internet; Diagnostics of the tendency to 13 types of addictions (G.V. Lozovaya); Test "Self-assessment of mental states" (G.Yu. Eysenck); Methodology "Diagnostics of typologies of psychological defense" (R. Plutchikas adapted by L.I. Wasserman, O.F. Eryshev, E.B. Klubovoy, etc.); 
Methodology for determining stress resistance and social adaptation of Holmes and Rage; $\mathrm{K}$. Young «Internet Addiction Test» and methods of mathematical statistics.

\section{Research results}

At the initial stage of the empirical study, we used the author's questionnaire to determine the amount of time spent by respondents on the Internet. We obtained the following results: $14 \%$ of respondents spend from 1 to 3 hours a day on the Internet, $43 \%$ of respondents spend from 3 to 6 hours a day on the Internet, $23 \%$ of respondents spend from 6 to 9 hours a day on the Internet, $20 \%$ of respondents spend from 9 hours or more on the Internet. Thus, the data obtained shows that about $50 \%$ of respondents spend up to 6 hours a day on the Internet (more than half of the working day).

It should be noted that the Internet has become a part of everyday life, work, an instrument of interpersonal communication and search, storage of information, many respondents can not imagine their life without the Internet and only a part is aware of all the dangers and risks that can manifest themselves with excessive spending time on the Internet. For many, the Internet has become a significant value in their lives and the main tool through which they make contact with the world around them.

At the second stage of the study, using the method of diagnosing the propensity to 13 types of addictions (G. V. Lozovaya), we studied the influence of the level of propensity to Internet addiction on the manifestation of other types of addictive behavior.

To divide the respondents into groups of interest, according to the level of propensity to Internet addiction, as well as to study the influence of propensity to it on the manifestation of various types of addictive behavior, we used this method. During the interpretation of this test, we obtained results about students with different levels of addiction to Internet addiction. In most cases, we observed the greatest differences between groups with low and high levels of addiction to Internet addiction.

Based on the data obtained, we can say the following: students with a high level of propensity to Internet addiction, there are high indicators for such propensities to addictive behavior as: alcohol, gaming, work, drug, smoking (nicotine), a healthy lifestyle and a general propensity to addictive behavior. Depending on the increase in the level of Internet addiction, these indicators will grow.

Then, using the test "Self-assessment of mental states" (G. Yu. Eysenck), we examined the psychological states of the respondents. During the interpretation of this test, we found that respondents with a high tendency to Internet addiction are much more likely to experience anxiety (indicator 11.5), for particularly small and insignificant reasons, compared with respondents with a low tendency to Internet addiction, who have an anxiety index of 7.9 and feel comfortable, safe, and do not experience anxiety states. The group of respondents with a high tendency to Internet addiction shows the highest indicators of frustration in comparison with the group of respondents with a low tendency to Internet addiction. People with a high tendency to Internet addiction are able to experience a sense of failure in meeting their needs and desires, low self-esteem. In these respondents, frustration is characterized by one of the most serious factors in their life, it can be determined by the pressure of their work, friends and family, and reflect the level of experience of failure in meeting needs and desires. Internet addicts can spend more time in the Internet space, where they are in a free and safe space, to get rid of feelings of frustration. Based on the data obtained during the interpretation of the aggressiveness index for groups with low, medium, and high propensity to Internet addiction, we saw that there is a strong difference in this indicator at all three levels of propensity to Internet addiction and a tendency to increase depending on the level of propensity to Internet addiction. To the greatest extent, individuals with a high and medium propensity to Internet addiction are 
dominated by such an indicator as aggression, that is, we can say that there is a degree of aggressive behavior in their daily lives. Students who are not prone to Internet addiction are less susceptible to aggressive behavior in comparison with students with a high propensity to Internet addiction. In the course of further interpretation, we obtained data that indicate that the level of rigidity of students with a high level of Internet addiction is higher than in groups with a low level of Internet addiction and less than in groups with an average level of Internet addiction.

Thus, the severity of mental states depends on the level of propensity to Internet addiction. There is a tendency to increase anxiety, frustration, and aggression with an increase in the level of addiction to Internet addiction. This suggests that students with a high propensity for Internet addiction are more likely to show anxiety, frustration, and aggression. Similarly, individuals with a low propensity for Internet addiction are characterized by a lower level of manifestation of these psychological states.

During the interpretation of the data according to the method "Diagnostics of typologies of psychological protection" (R. Plutchik in the adaptation of L. I. Wasserman, O. F. Eryshev, E. B. Klubova, etc.), we saw that groups with different levels of propensity to Internet addiction have significant differences in the indicators of the severity of psychological protection (Fig. 1). We can say that the group with a high propensity for Internet addiction has pronounced psychological defenses such as denial, suppression, compensation, projection, substitution, intellectualization, and reactive formations.

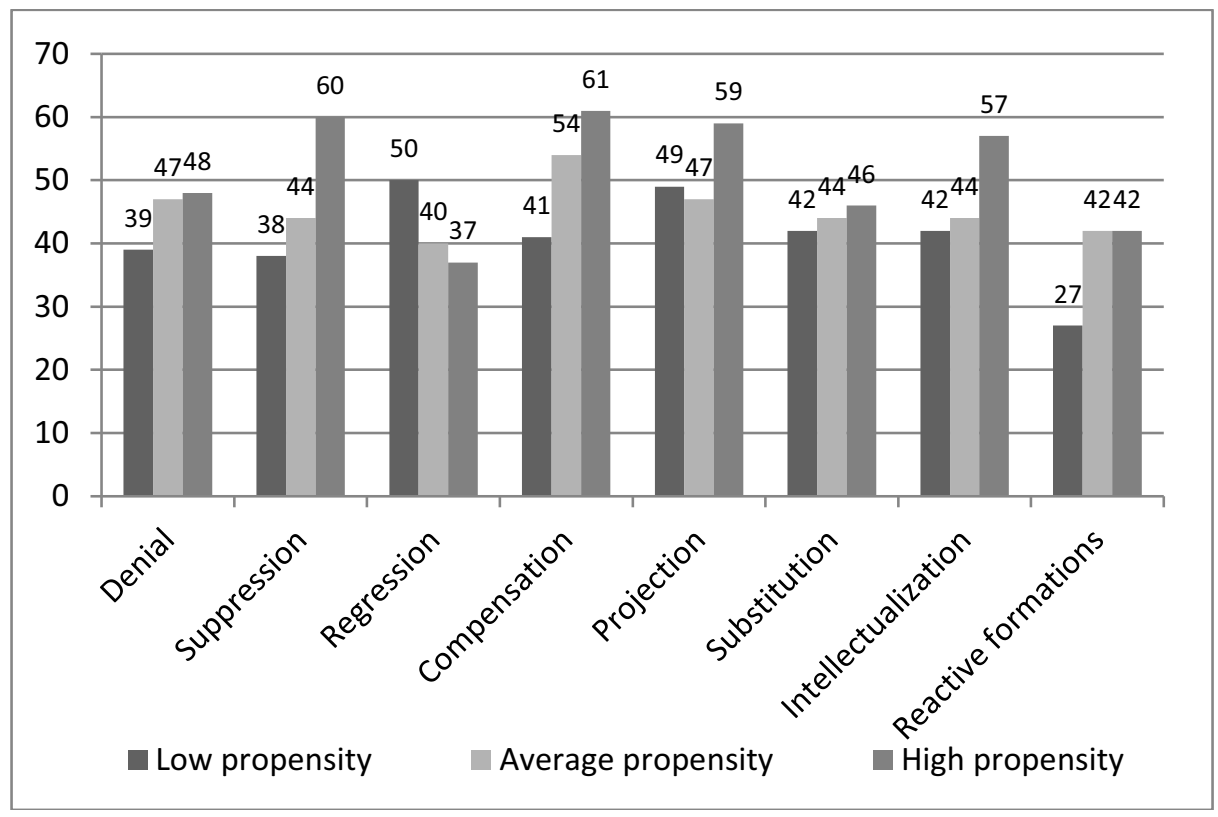

Fig. 1. The level of severity of psychological defenses of students with different levels of Internet addiction (in points).

Students with a high level of Internet addiction are more likely to involuntarily repress impulses and unacceptable thoughts into the unconscious and to display compensatory activities related to Internet use. We can observe the replacement of real communicationthe Internet, as well as other aspects of activity that can be connected with the computer and the Internet can also be compensated for, as well as aspects of interaction with the outside world, preference in these cases will be given to the most comfortable tools, namely gadgets, computer and the Internet. 
Students with a high level of Internet addiction are characterized by the suppression of emotions, such as hostility and anger, which are directed at objects that are less dangerous or more accessible than those that caused negative emotions and feelings. The severity of the projection in the respondents indicates that the respondents attribute their own thoughts, feelings, motives, character traits, to someone or something, believing that they have perceived something coming from outside, and not from within themselves, as well as a tendency to unconsciously try to abstract from their feelings. In this case, projection manifests itself in increased curiosity about other people's lives, judgment, or copying a lifestyle. Such psychological defenses as compensation, denial, suppression, projection, substitution, intellectualization, reactive formations tend to increase in severity with an increase in the level of propensity to Internet addiction. That is, the severity of these psychological defenses may directly depend on the level of Internet addiction.

So, it can be stated that the level of Internet addiction can affect the severity of such psychological defenses as denial, suppression, compensation, projection, substitution, intellectualization and reactive formations. The data obtained by us indicates that the higher the level of propensity to Internet addiction, the stronger these psychological defenses will be expressed, that is, the severity of these psychological defenses depends on the level of Internet addiction.

In the course of interpreting the methodology for determining stress resistance and social adaptation of Holmes and Rage, we obtained results indicating that students with a high level of propensity to Internet addiction are less stress-resistant and socially adapted compared to a group of students with a low level of Internet addiction. It should also be noted that, depending on the level of propensity to Internet addiction, there will be changes in the level of stress resistance and social adaptation of students. With an increase in the level of propensity to Internet addiction, stress resistance and the level of social adaptation will decrease.

Thus, the level of propensity to Internet addiction can affect the level of resistance to stress and social adaptation of students. The data obtained indicate that with an increase in the level of Internet addiction, students ' level of stress tolerance will decrease. It should be noted that in this method, the higher the indicator, the lower the level of resistance to stress and social adaptation.

To further analyze the results for a group of students with a high level of Internet addiction, we used the Kimberly Young «Internet Addiction Test». Based on the data we have obtained, we can say that students with a high level of Internet addiction have problems using the Internet $77 \%$, are regular Internet users $9 \%$ and are Internet addicts $14 \%$ of students with a high level of Internet addiction. Based on the above, we can say that students with a high level of propensity to Internet addiction can have a full-fledged addiction, in our case this is $14 \%$ of the group with a high propensity to Internet addiction and $77 \%$ have problems, which is a significant indicator.

So, we can state the fact that students with a high level of propensity for Internet addiction may have signs of addictive behavior, as well as formed addiction, with all the ensuing consequences.

\section{Discussion}

In our understanding of the essence of this problem, the research was conducted by A. Weinstein, who identified three subtypes of Internet addiction: excessive games, email/text messages, and sexual hobbies. Based on the research conducted among students from different countries, the author identified the following four components regarding Internet addiction: 1) excessive use, often associated with a loss of sense of time and focus of the main drive, 2) withdrawal, including feelings of anger, tension, and/or depression when the 
computer is unavailable, 3) tolerance, including increased computer hardware, software, or several hours, and 4) adverse effects, including arguments, lies, poor achievement, social isolation, and fatigue $[14,15]$.

Within the framework of modern realities, it is debatable whether Internet addiction is an independent diagnosis or rather the product of other existing disorders, such as depression, anxiety, ADHD, or pattern control disorders [1,3,6,7,8,13,14,15,16,17]. A fairly large number of authors believe that Internet addiction is a behavioral addiction $[15,17]$. However, the pathophysiological mechanisms underlying Internet addiction are still under investigation.

A number of studies on the treatment of Internet addiction can be noted, which are based on interventions and strategies used in the treatment of disorders associated with the use of psychoactive substances $[1,3,6,7,8,13,14,15,16,17,19,20,22]$.

Summarizing the presented data, we can say that the essence of Internet addiction is approached in different ways. There are different approaches to its treatment, and there are actually several effective methods of therapy.

\section{Conclusion}

Using the methods of mathematical statistics, we found that there is a significant relationship between Internet addiction and alcohol, labor, drug, nicotine, and the general tendency to addictive behavior.

The level of propensity to Internet addiction is associated with the manifestation of such addictions as alcohol, labor, drug, nicotine and general propensity to addictive agents. The higher the level of propensity for Internet addiction, the higher the level of propensity for these types of addictive behavior. The most reliable indicators of dependence are the relationship between labor-addiction (at $\mathrm{p}<0.05, \mathrm{r}=0.51$ ) and labor-addiction (at $\mathrm{p}<0.05$, $\mathrm{r}=0.42$ ). There is a direct, medium-close relationship between Internet addiction and alcohol addiction, and a moderate, direct-close relationship between Internet addiction and work addiction.

A direct, moderate close relationship is seen between a high level of propensity to Internet addiction and nicotine addiction (at $\mathrm{p}<0.05, \mathrm{r}=0.41$ ), drug addiction (at $\mathrm{p}<0.05$, $\mathrm{r}=0.37$ ), and general propensity to addictive agents (at $\mathrm{p}<0.05, \mathrm{r}=0.39$ ). In this case, the most significant relationship between the level of addiction to Internet addiction and nicotine addiction, less pronounced relationship between Internet addiction and such addictions as drug and general. Based on the data obtained, we can talk about the following:

There is a significant correlation between the level of propensity to Internet addiction in students and the manifestation of alcohol, labor, drug, nicotine and general addiction. In this case, a stronger correlation is observed between the propensity to Internet addiction and alcohol addiction, to a lesser extent, the propensity to Internet addiction has an impact on labor, nicotine, and the overall level of propensity to addictive behavior.

When analyzing the data on the correlation analysis between a high propensity to Internet addiction and the manifestation of psychological defenses, we found a significant relationship, an average closeness between Internet addiction and compensatory activity (at $\mathrm{p}<0.05, \mathrm{r}=0.52$ ), as well as direct, moderate relationships between a high level of propensity to Internet addiction and suppression (at $\mathrm{p}<0.05, \mathrm{r}=0.45$ ) and Internet addiction and Intellectualization (at $\mathrm{p}<0.05, \mathrm{r}=0.37$ ). A more significant relationship is seen between the tendency to Internet addiction and compensation, To a lesser extent, there is a correlation between the tendency to Internet addiction and suppression, and between intellectualization and the tendency to Internet addiction, but the indicator is lower than between the tendency to Internet addiction and such psychological defenses as compensation and suppression. 
The data obtained by us on the indicators of the test "Self-assessment of mental states" (G. Y. Eysenck) are also confirmed. We obtained reliable indicators of interdependence, direct, average closeness between anxiety and propensity to Internet addiction (at $\mathrm{p}<0.05$, $\mathrm{r}=0.52$ ), direct, moderate closeness of the relationship is seen between propensity to Internet addiction and aggression (at $\mathrm{p}<0.05, \mathrm{r}=0.42$ ), therefore, we can say that the propensity to Internet addiction is directly proportional to anxiety and aggression, and therefore there is a relationship between Internet addiction and anxiety, aggression in students and the more pronounced Internet addiction is, the greater the severity we can observe in anxiety. Thus, we prove the relationship between the tendency to Internet addiction and the level of anxiety and aggression.

When analyzing the data on the correlation analysis between the propensity to Internet addiction and Internet addiction and the Level of resistance to stress, we obtained the following indicators: Between the level of propensity to Internet addiction and Internet addiction, a direct, average closeness of the relationship is seen (at $\mathrm{p}<0.05, \mathrm{r}=0.59$ ) and between the propensity to Internet addiction- addiction and the level of resistance to stress, we obtained a direct, moderate tightness of the relationship (at $\mathrm{p}<0.05, \mathrm{r}=0.42$ ), The data obtained by us indicate that the level of propensity to Internet addiction correlates with fullfledged Internet addiction, and we also found a relationship between the propensity to Internet addiction and the level of stress in students.

Summarizing the data obtained, we can conclude that 1) there is a relationship between the level of psychological states (aggression, anxiety) of students and the level of propensity to Internet addiction. 2) The level of propensity to Internet addiction can affect the level of severity of psychological defenses (suppression, compensation, intellectualization). 3) There is a relationship between the level of addiction to Internet addiction and the level of stress tolerance.

\section{References}

1. I. Abakumova, Z. Brizhak, A. Kukulyar, A. Kolenova, E3S Web of Conferences 210, 19003 (2020). doi.org/10.1051/e3sconf/202021019003.

2. V.A. Burova, Internet addiction as a form of non-chemical adaptive disorders (Novosibirsk, 2014).

3. Z.I. Brizhak, A.S. Kolenova, A.M. Kukulyar, International Journal of Medicine and Psychology 4, 78-84 (2021).

4. F. Ghamari, A. Mohammadbeigi, N. Mohammadsalehi, A.A. Hashiani, Indian Journal of Psychological Medicine 33(2), 158-62 (2011).

5. I. Goldberg, Internet addiction disorder, (1996). http://www.psycom.net, last accessed 2021/01/09.

6. C. Gregory, Internet Addiction Disorder. https://www.psycom.net/iadcriteria.html, last accessed 2021/02/10.

7. D.H. Han, P.F. Renshaw, Journal of Psychopharmacology 26(5), 689-96 (2012).

8. A.S. Kolenova, A.M. Kukulyar, N.N. Bessonova, N.A. Gudz, International Journal of Medicine and Psychology 4, 38-44 (2021).

9. R. Poli, E. Agrimi, Nordic Journal of Psychiatry 66(1), 55-9 (2012).

10. V. Sysoev, B.I. Lebedev, T.P. Filatova, ISOM 4(1), 97-109 (2016).

11. A.N. Teslenko, CSW 5(124), (2017). https://cyberleninka.ru/article/n/molodezhnayainternet-addiktsiya-kak-ekzistentsionalnaya-problema, last accessed 2020/12/27.

12. S.N. Varlamova, E.R. Goncharova, I.V. Sokolova, Monitoring 2(125), 165-182 (2015). 
13. A.E. Voiskunsky, Humanistic research on the Internet, Mozhaysk-Terra. 100-131 (2000)

14. A.Weinstein, Med. psihol. Ross 4 (33), 3 (2015). http://mprj.ru, last accessed 2021/02/13.

15. A. Weinstein, L.C. Feder, K.P. Rosenberg, P. Dannon, Internet Addiction Disorder: Overview and Controversies. http://scitechconnect.elsevier.com/wpcontent/uploads/2014/10/Internet-Addiction-Disorder.pdf, last accessed 2021/02/10.

16. A. Weinstein, D. Dorani, R. Elhadif, Y. Bukovza, A. Yarmulnik, P. Dannon, Annals of Clinical Psychiatry 27(1), 4-9 (2015).

17. K.S. Young, Internet Addiction: Symptoms, Evaluation, And Treatment. http://netaddiction.com/articles/symptoms.pdf, last accessed 2021/02/10.

18. K.S.Young, Med. psihol. Ross 4(33), 2 (2015). http://mprj.ru, last accessed 2021/02/13.

19. J.Y. Yen, C.F. Yen, H.Y. Wu, C.J. Huang, C.H. Ko, Cyberpsychology Behavior and Social Networking 14(11), 649-55 (2011).

20. J. Xu, L.X. Shen, C.H. Yan, H. Hu, F. Yang, L. Wang, S.R. Kotha, L.N. Zhang, X.P. Liao, J. Zhang, F.X. Ouyang, J.S. Zhang, X.M. Shen, BMC Public Health 12(1), 1106 (2012).

21. O.V. Zavalishin, N.A. Zagumennov, E.S. Postoeva, Scientific journal of Kuban state agrarian University - Scientific Journal of KubSAU 105 (2015). http://cyberleninka.ru/article/n/internet-addiktsiya-odna-iz-aktualnyh-problem sovremennosti, last accessed 2020/12/13.

22. F. Zanetta Dauriat, A. Zermatten, J. Billieux, G. Thorens, G. Bondolfi, D. Zullino, Y. Khazaal, European Addiction Research 17(4), 185-9 (2011). 